\title{
Phase diagram senses orbitals and prompts a fundamental constant
}

Shapiullah Abdulvagidov ( $\square$ abdulvagidovshb@gmail.com )

Russian Academy of Sciences

\section{Research Article}

Keywords: condensed-matter, phase diagram, intermolecular attraction

Posted Date: September 10th, 2021

DOI: https://doi.org/10.21203/rs.3.rs-885509/v1

License: (9) This work is licensed under a Creative Commons Attribution 4.0 International License. Read Full License 


\title{
Phase diagram senses orbitals and prompts a fundamental constant
}

\author{
Shapiullah Belalovich Abdulvagidov
}

Institute of Physics of Dagestan Federal Research Centre of RAS, Makhachkala 367015 Republic of

Dagestan, Russian Federation

abdulvagidovshb@gmail.com

Van der Waals' discovery of that the volumes of molecules and their intermolecular attraction between them cause the peculiarities of the phase diagrams of gases and liquids ${ }^{1}$ gave the greatest impact on the progress of science and industry.

Unfortunately, the phase charts of solids capable to advance scientific and technical progress remain uncomprehended mystery. Only the certain linear phase boundaries are understood by the struggle of magnetic field $B$ against the thermal agitation $^{2,3}$. Here we show that the intersection volume of internal atomic orbitals determines the form of phase boundary and, furthermore, energy per unit volume of the intersection is a new fundamental constant $v=8.941 \mathrm{eV} / \AA^{3}$. Together with the known struggle contribution ${ }^{2,3}$ to $T_{\mathrm{C}}(B)$, we found a term proportional to the intersection volume of $3 d e_{g}$ and $2 p$ orbitals in the $\mathrm{Sm}_{0.55} \mathrm{Sr}_{0.45} \mathrm{MnO}_{3}$ manganite. Hysteresis of $\boldsymbol{T}_{\mathrm{C}}$ is described by the avalanche-like widening of the intersection volume due to reducing the Coulomb distortion with double-exchange ferromagnetism. The pressure- $T_{C}$ diagram $^{4}$ of $\left(\mathrm{Sm}_{1-x} \mathrm{Nd}_{x}\right){ }_{0.55} \mathrm{Sr}_{0.45} \mathrm{MnO}_{3}(x=0,0.2$, $0.4,0.5)$ is approximated with the same parameters as the $T_{C}(B)$ diagram of $\mathrm{Sm}_{0.55} \mathrm{Sr} 0.45 \mathrm{MnO}$. Furthermore, the diamond's melting point $4157^{\circ} \mathrm{C}$ calculated from the intersection volume of $s p^{3}$-orbitals is in excellent agreement with the real

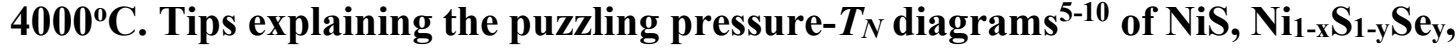
$\mathrm{BaVS}_{3}, \mathrm{~V}_{2} \mathrm{O}_{3}, \mathrm{RNiO}_{3}$ and ferrites were given. Our discovery is the beginning of condensed-matter geometrodynamics and marks an era of studying phase diagrams to advance condensed-matter physics and tailor new materials with predicted properties necessary in sunrise industries. Moreover, internucleon, interquark and intergluon orbital intersections would be useful for understanding the properties of nuclei, nucleons and quarks.

Understanding the phase diagram of solid avails us the nature of interatomic interaction inducing the phase transition, but phase-transition lines often behave intriguing manner. It is especially interesting the phase chart of organic and cuprate superconductors where four different states meet at multicritical point ${ }^{11}$. The phase diagram of iron, however, is no less attractive and puts up knotty issue about whether the number of solid phases should be raised to five ${ }^{12}$.

Puzzling over phase diagrams of gases Van der Waals came to that the volume of a molecule of the gas had the matter and they interacted between them with the force named later in his honour ${ }^{1}$. He also asked himself how the solid state behaved, however, was silent on this question. Atoms of inert gases have completely closed electron shells, and molecules of diatomic gases the closed valent shells at least, so Van der Waals' 
liquid-gas equation state is in fact the consequence of dispersion forces between the closed shells very hard of Pauli principle. Emphasizing the great impact of Van der Waals' findings, H. Kamerlingh Onnes ${ }^{13}$ wrote in 1910 'that Van der Waals' studies have always been considered as a magic wand for carrying out experiments and that the Cryogenic Laboratory at Leyden has developed under the influence of his theories". Liquefying helium allowed H. Kamerlingh Onnes in 1911 discovered superconductivity, so unravelling the mystery of phase charts of liquefiable gases had moved forward physics not only gases and liquids, but also solids.

The aim of this article is to reveal the nature of the phase diagram of solid, that is, to answer the question been silent by Van der Waals. We have asked ourselves why the $T_{\mathrm{C}}(B)$ diagram of $\mathrm{Sm}_{0.55} \mathrm{Sr}_{0.45} \mathrm{MnO}_{3}$, the most-studied composition, behave straightly with different slopes along the $B C$ and the $D E$ lines and curvedly on the segment $A C$ (Fig. 1).

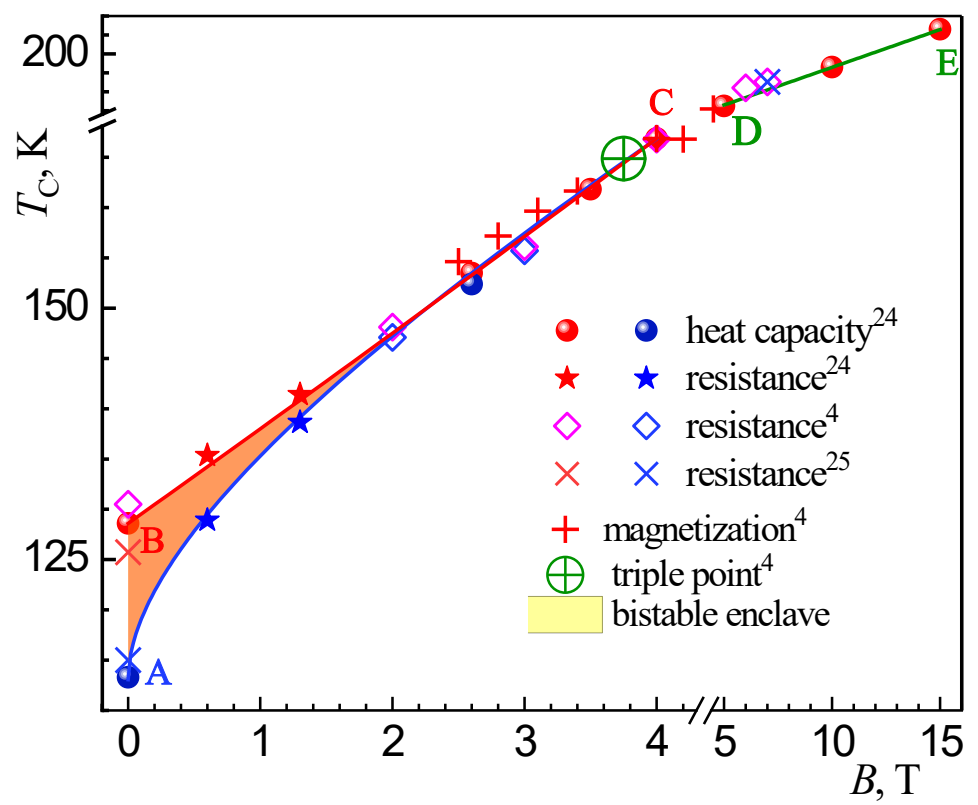

Fig. 1. $\mid$ The $\mathrm{Sm}_{0.55} \mathrm{Sr}_{0.45} \mathrm{MnO}_{3} T_{\mathrm{C}}(B)$ diagram. The ferromagnetism appears at downward and/or rightward crossing of the curve $A C$ but disappears at upward and/or leftward crossing of the line $B C$. So if the bistable region has been reached from under $A C$ (above $B C$ ) it is ferromagnetic (paramagnetic), that is, the nonvolatile memory effect driven both by temperature and by magnetic field takes place. $A C, B C$ and $D E$ are seen to excellently describe the Curie points (the red signs at heating and the blue at cooling) of polycrystallines ${ }^{24,25}$ and single crystal ${ }^{4}$.

The $D E$-line energy slope $\frac{k_{\mathrm{B}}}{\mu} \Delta T_{\mathrm{C}} / \Delta B=0.99$ (according to Hund's rules $\mu=6 \mu_{\mathrm{B}}$ both for $\mathrm{Mn}^{+3}$ and $\mathrm{Mn}^{+4}$ ) means that magnetic field only suppresses the thermal agitation and so the Curie point is linear:

$$
T_{\mathrm{C}}=\frac{\mu}{k_{B}} B \cos \theta,
$$

$\theta$ is an angle between the Mn magnetic moment and the magnetic field $\boldsymbol{B}$ or, at the same time, a tilting angle between the adjacent $\mathrm{MnO}_{6}$ octahedrons. The $D E$-line energy slope 
gives tilting angle $\theta_{D E}=\arccos 0.99=8.2^{\circ}$. The question, which could be answered by future studies, arises how long such linearity will remain above $15 \mathrm{~T}$.

Our attempts to interpret the steep energetic pitch 2.37 of $B C$ as easy as $D E$ failed. It appeared to reveal the secret of $B C$ was previously necessary to interpret $A C$ and the zero-field hysteresis $A B$, which is a passway to $B C$. The insight has come with a lightning speed when we saw a spheric cap in a stereometry textbook, a portion of a sphere cut off by a plane, with the volume $V=\pi h^{2}(R-h / 3), h$ is an intersection height and $R$ a radius of the sphere. We supposed that the transition temperature $T_{t r}$ is proportional to the volume $2 \mathrm{~V}$ of the double spheric cap formed at the intersection of the orbitals (Fig. 2a) with the coefficient $v$ being a volumetric energy density:

$$
T_{t r}=\frac{2 v}{k_{\mathrm{B}}} \pi h^{2}(R-h / 3) .
$$

This formula is suited for different transitions because the subscript ' $t r$ ' means Curie, Neel or melting point for ferromagnetic, antiferromagnetic or melting transition, accordingly.
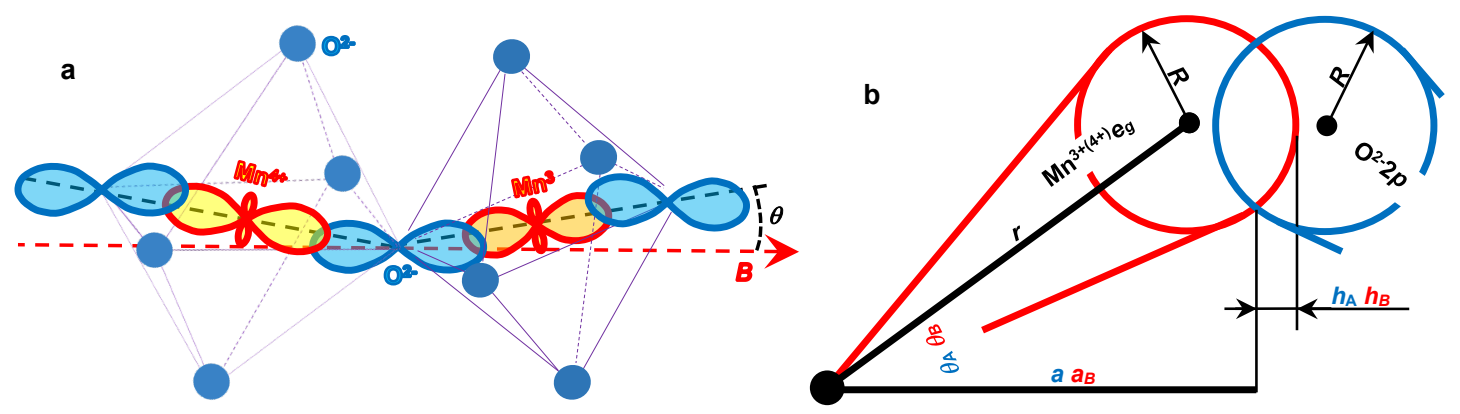

Fig. 2. The biconvex-lens volume of the intersection of the orbitals $\mathrm{Mn}^{3+(4+)} 3 d e_{g}$ and $\mathbf{O}^{2-2} \boldsymbol{p}$, and geometric calculation of its intersection height $\boldsymbol{h}_{\boldsymbol{B}}$. a, The tilting angle $\theta$ between $\mathrm{MnO}_{6}$ octahedrons and magnetic field $\boldsymbol{B}$ determines the magnetic energy $6 \mu_{\mathrm{B}} \cos \theta$ of the $\mathrm{Mn}^{3+(4+)}$ cations. b. The blue letterings belonging to the point $A$ yield the distance $r$ from the site $\mathrm{Mn}^{3+(4+)}$ or $\mathrm{O}^{2-}$ to the center of the curvature $R$ of the spheric end of its $e_{g}$ or $2 p$ orbital, $r=\left(a-R+h_{A}\right) / \cos \theta_{A}$. The red letterings belonging to the point $B$ give $h_{B}=r \cos \theta_{B}-a_{B}+R$, which with the $r$ calculated from the blue lettering, transforms into Exp. (4).

Obviously, the intersection height $h=\varepsilon a$; $a$ is half a distance between $\mathrm{Mn}^{3+}$ or ${ }^{4+}$ and $\mathrm{O}^{2-}$ and $\varepsilon$ a relative striction. If a solid shrinks in magnetic field, the magnetostatic energy of a transition-metal cation spends on to enhance the elastic energy a unit cell, that is, $\mu \cdot \boldsymbol{B}=V E \varepsilon^{2} / 2 ; E$ is a Young's modulus and here $V$ a unit-cell volume. Then $\varepsilon=$ $\sqrt{2 \mu \cdot \boldsymbol{B} / V E}$ and so $h=a \sqrt{2 \mu \cdot \boldsymbol{B} / V E}$. On $A C h_{A C}=a \sqrt{2 \mu \cdot \boldsymbol{B} / V E}+h_{A}, h_{\mathrm{A}}$ is added on account of a zero-field intersection height inducing the zero-field Curie point at the point $A$. Inserting $h=h_{A C}$ into Exp. (2) gives

$$
T_{\mathrm{C}}=\frac{2 \pi v}{k_{\mathrm{B}}}\left(\frac{2 a^{2} \mu \cdot \boldsymbol{B}}{V E}+2 h_{A} a \sqrt{\frac{2 \mu \cdot \boldsymbol{B}}{V E}}+h_{A}^{2}\right)\left[R-\left(a \sqrt{\frac{2 \mu \cdot \boldsymbol{B}}{V E}}+h_{A}\right) / 3\right] .
$$


Magnetic field supports ferromagnetism along $A C$ same as on $D E$, therefore, the best physical description of $A C$ is the sum of Exps. (3) and (1) at $\cos \theta$ linearly turning down from $\theta_{A}$ at point $A$ to $\theta_{C}$ at the point $C$ (the linearization is quite reasonable as $\cos \theta$ also linearly depends on temperature ${ }^{14}$ and the radius ${ }^{15}$ of the rare-earth ion). The rotation $\theta_{A}-\theta_{C}$ also equals a sum of those at the zero-field hysteresis $A B$ and along $B C$;

otherwise, $A C$ and $B C$ would not flow at the triple point $C$ together. We found the best fit with the root-mean-square error $0.58 \mathrm{~K}$ at the corresponding parameters in the Table. So enigmatic behaviour of $A C$ is de-facto a steric effect - the field-induced overlapping of the orbitals $3 \mathrm{~d} e_{\mathrm{g}}$ and $2 p$.

At $h=h_{\mathrm{A}}$ Exps (2) and (3) give the zero-field cooling Curie point $T_{C}^{A}=113.06 \mathrm{~K}$. A reason question suggests itself why the zero-field heating Curie point $T_{C}^{B}$ is higher than $T_{C}^{A}$. The issue was qualitatively discussed elsewhere ${ }^{16}$. By resuming, we have come to the following quantitative explanation. The initial overlap $h_{\mathrm{A}}$ triggers double exchange which, in turn, due to extra removal of the Coulomb distortion ${ }^{16}$, inflates the orbital overlapping to $h_{\mathrm{B}}$, the Curie point heightening to $T_{C}^{B}$. The geometric constructions for points $A$ and $B$ (Fig. 2b) gives

$$
h_{B}=\left(a-R+h_{A}\right) \cos \theta_{B} / \cos \theta_{A}-a_{B}+R .
$$

Exp (2) at $h=h_{\mathrm{B}}$ yields $T_{C}^{B}=129.99 \mathrm{~K}$ (calculated from the entries for the point $B$ in the Table) in excellent consistent with the experimental $128.60 \mathrm{~K}$. Thus, at $T_{C}^{A}$ the threshold intersection $h_{\mathrm{A}}$ avalanche-like widens till $h_{\mathrm{B}}$ generating $T_{C}^{B}$. It is natural to observe $T_{C}^{B}$ may be at heating only. Such is the mystery of the hysteresis taking place at the firstorder-like phase transition.

Now we can interpret $B C$, wherein magnetic field orientates the $\mathrm{MnO}_{6}$ octahedrons and straightens $\theta$ from $\theta_{B}$ to $\theta_{C}$, the intersection expanding. As $\theta$ does not change a spacing between the $\mathrm{Mn}^{3+}$ or ${ }^{4+}$ and $\mathrm{O}^{2-}$ ions, for $B C$ Exp. (4) transforms into

$$
h_{B C}=\left(a_{B}-R+h_{B}\right) \cos \theta / \cos \theta_{B}-a_{B}+R,
$$

where $\cos \theta \in\left[\cos \theta_{B} ; \cos \theta_{C}\right]$ is linear of the field. Magnetic field supports magnetism on $B C$ same as on $A C$ and $D E$. And the best physical description of $B C$ with the root-meansquare error $1.24 \mathrm{~K}$ is a sum of Exp. (2) with $h=h_{B C}$ and $\operatorname{Exp}$ (1) (see the Table for the fit parameters).

Our supposition confirmed on other cases. In the case of the $T_{t r}(P)$ diagram, the Hooke law $P=E(P) \varepsilon$ (the Young's modulus is supposed to depend on pressure as well as temperature ${ }^{17}$, magnetic order ${ }^{18}$ and even form $\left.{ }^{19}\right)$ gives $h=a P / E(P)+h_{0}\left(h_{0}\right.$ is an intersection height at the normal conditions) which turns Exp. (2) into

$$
T_{t r}=\frac{2 \pi v}{k_{\mathrm{B}}}\left(\frac{a^{2} P^{2}}{E(P)^{2}}+2 h_{0} \frac{a P}{E(P)}+h_{0}^{2}\right)\left[R-\left(\frac{a P}{E(P)}+h_{0}\right) / 3\right] .
$$

This expression excellently describes The $T_{\mathrm{C}}(P)$ diagram $^{4}$ of $\left(\mathrm{Sm}_{1-x} \mathrm{Nd}_{x}\right)_{0.55} \mathrm{Sr}_{0.45} \mathrm{MnO}_{3}$ $(x=0,0.2,0.4,0.5)$ (see Fig. 3 and the Table).

In the case of superexchange, our discovery let us know the nature of the antiferromagnetism ${ }^{5-7}$ not clear for decades in $\mathrm{NiS}_{2} \mathrm{~V}_{2} \mathrm{O}_{3}$ and $\mathrm{RNiO}_{3}(\mathrm{R}=\mathrm{Pr}, \mathrm{Nd}$, $\mathrm{Nd}_{0.7} \mathrm{La}_{0.3}$ ). The pressure-induced shift of the transition-cation planes with respect to the anion planes causes the $3 \mathrm{~d} e_{\mathrm{g}}$ orbital to hold away from the $2 p$ orbital and so their intersection narrows then $T_{N}$ lowers and the $e_{\mathrm{g}}$ electrons, which contribute to the 
antiferromagnetism and cannot migrate as current carriers, gradually delocalize that leads to metallization.

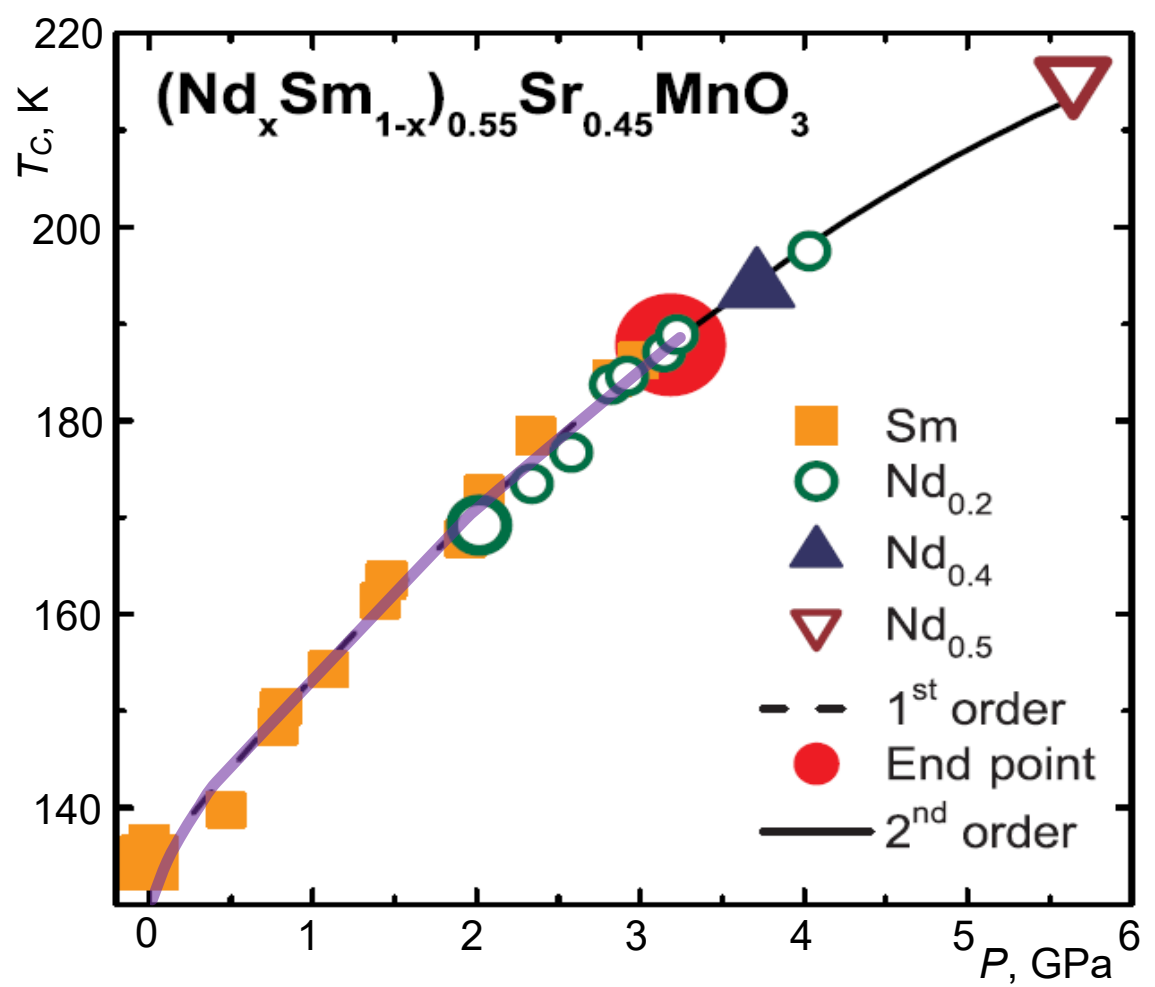

Fig. 3. | The $\boldsymbol{T}_{\mathrm{C}}(P)$ diagram reproduced of ${ }^{4}$ fitted by Exp. (6) at the nonlinear Young modulus $E(P)=325 P^{1 / 3}+E_{0}$ and $h_{0}=h_{\mathrm{B}}$ (the translucent purple curve).

The $T_{N}(P)$ diagram ${ }^{10}$ of $\mathrm{BaVS}_{3}$ become understandable if to take into account the increase of the orbital overlapping in the V-S chains, which favours to antiferromagnetism but forbids Mott's conductance. The linear $T_{N}(P)$ of the orthoferrites ${ }^{9}$ $\mathrm{Gd}, \mathrm{Pr}$ and Tm means the fulfillment of Hooke law at the small rare-earth-ion radius. The more rare-earth-ion radius in the La orthoferrite ${ }^{9}$ shortens the $\mathrm{Fe}-\mathrm{O}$ bond and so widens the intersection of the orbitals $\mathrm{Fe}^{3+} 3 d$ and $\mathrm{O}^{2-} 2 p$ that causes the lift and nonlinearity of the $T_{N}(P)$. Exp. (6) with the internal chemical pressure $P_{i} \sim 1-y / y_{p}\left(y_{p}\right.$ is a doping index of the paramagnetic composition) instead of $P$ could fit the temperature-doping phase diagram of the antiferromagnet ${ }^{8} \mathrm{Ni}_{1-\mathrm{x}} \mathrm{S}_{1-\mathrm{y}} \mathrm{Se}_{\mathrm{y}}$.

Finally, our calculation of diamond's melting-point is the brightest approbation of our condensed-matter geometrodynamics. The diamond's $s p^{3}$-orbitals intersection is the carbon's $p$-orbital length minus the carbon's covalent radius ${ }^{20}$ in diamond: $h=r_{p}-r_{\text {dia }}^{c o v}$. The $r_{p}$ is obtained as the carbon-hydrogen distance in methane plus the hydrogen covalent radius and minus Bohr radius: $r_{p}=r_{\mathrm{CH}_{4}}+r_{\mathrm{H}}^{\text {cov }}-r_{B}$. Then $h=1.087+0.31-$ $0.529177-0.772=0.095823 \AA$ A . Exp. (2) with this $h$ and $R=r_{p} / 4$ multiplied by the four covalent bonds gives the diamond's melting point $4157^{\circ} \mathrm{C}$ in excellent agreement with the real $4000^{\circ} \mathrm{C}$.

Thus, the phase diagram of the solid has been completely interpreted. It turned out that its certain phase boundaries sense the intersection of intra-atomic orbitals. 
Intersection volume has geometric energy whose volumetric density is a new

fundamental constant. Thus, the steric effect well-known in condensed-matter physics has at last received its quantitative explanation. The phase diagram of solid surprisingly appeared a bridge between the classic and the quantum physics, allowing us to get the precise picture of the orbital intersection unreachable even in the neutron microscopy.

\section{References}

1. Van der Waals, J. D. The equation of state for gases and liquids. Nobel Lecture, December 12, 1910. https://www.nobelprize.org/prizes/physics/1910/waals/lecture/.

2. Feng, J. et al. Large linear magnetoresistance in Dirac semimetal $\mathrm{Cd}_{3} \mathrm{As}_{2}$ with Fermi surfaces close to the Dirac points. Phys. Rev. B 92, 081306 (2015).

3. Samatham, S., Barua, S. \& Suresh, K.G. Spin-flop quasi-first order transition and putative tricritical point in $\mathrm{Gd}_{3}$ Co. JMMM 4444, 439-443 (2017).

4. Demko, L. et al. Multicritical end point of the first-order ferromagnetic transition in colossal magnetoresistive manganites. Phys. Rev. Lett. 101, 037206 (2012).

5. McWhan, D.B., Marezio, M., Remeika, J. P. \& Dernier, P.D. Pressure-temperature phase diagram and crystal structure of NiS. Phys. Rev. B 5, 2552-2555 (1972).

6. Carter, S.A., Rosenbaum, T.F., Lu, M. \& Jaeger, H.M. Magnetic and transport studies of pure $\mathrm{V}_{2} \mathrm{O}_{3}$ under pressure. Phys. Rev. B 49, 7898-7903 (1994).

7. Obradors, $X$. et al. Pressure dependence of the metal-insulator transition in the chargetransfer oxides $\mathrm{RNiO}_{3}\left(\mathrm{R}=\mathrm{Pr}, \mathrm{Nd}, \mathrm{Nd}_{0.7} \mathrm{La}_{0.3}\right)$. Phys. Rev. B 47, 12353-12356 (1993).

8. Matoba M., Anzai, S. \& Fujimori, A. Thermal expansion, thermoelectric power, and XPS study of the non-metal-metal transition in $\mathrm{Ni}_{1-\mathrm{x}} \mathrm{S}_{1-\mathrm{y}} \mathrm{Se}_{\mathrm{y}}$.J. Phys. Soc. Jap. 60, 4230-4244 (1991).

9. Halasa, N.A., DePasquali, G. \& Drickamer, H.G. High-pressure studies on ferrites. Phys. Rev. B 10, 154-164 (1974).

10. Fazekas, P. et al. Magnetic-field-induced transition in BaVS 3 . Phys. Rev. B 75, 035128 (2007).

11. McKenzie, R.N. Similarities between organic and cuprate superconductors. Science 278, 820-821 (1997).

12. Anderson, O.L. Iron: beta phase frays. Science 278, 821-822 (1997).

13. J. D. van der Waals, J. D. - Biographical. https://www.nobelprize.org/prizes/physics/1910/waals/biographical/.

14. Medarde, M. et al. High-pressure neutron-diffraction study of the metallization process in $\mathrm{PrNiO}_{3}$. Phys. Rev. B 52, 9248-9258 (1997).

15. Zhou, J.-S. \& Goodenough, J.B. Unusual evolution of the magnetic interactions versus structural distortions in $\mathrm{RMnO}_{3}$ perovskites. Phys. Rev. Lett. 96, 247202 (2006).

16. Abdulvagidov, S.B., Djabrailov, S.Z. \& Abdulvagidov, B.S. Nature of novel criticality in ternary transition-metal oxides. Sci Rep 9, 19328 (2019). https://doi.org/10.1038/s41598-019-55594-w

17. Lalitha, G. \& Reddy, P.V. Elastic behavior of some manganite-based orthorhombic $\mathrm{RMnO}_{3}(\mathrm{R}=\mathrm{Sm}, \mathrm{Eu}, \mathrm{Gd}$, Dy) multiferroics. JMMM 320, 754-759 (2008).

18. Street, R. \& Lewis, B. Anomalous variation of Young's modulus of antiferromagnetics at the Neel point. Nature 168, 1036-1037 (1951). 
19. Treacy, M.M.J., Ebbesen, T.W. \& Gibson, J.M. Exceptionally high Young's modulus observed for individual carbon nanotubes. Nature 381, 678-680 (1996).

20. Pyykko, P. Refitted tetrahedral covalent radii for solids. Phys. Rev. B 85, 035128 (2012).

21. Kurbakov, A.I., Trunov, V.A. \& Andre, G. Study of effect isotopic substitution ${ }^{16} \mathrm{O} \rightarrow{ }^{18} \mathrm{O}$ in $\mathrm{Sm}_{1-\mathrm{x}} \mathrm{Sr}_{\mathrm{x}} \mathrm{MnO}_{3}$-type $(\mathrm{x}=0.45$ and 0.50$)$ manganites by powder neutron diffraction. Crystallography Reports 49, 899-906 (2004).

21. Sommerfeld, A. Atombau und spektrallinien (Friedr. Vieweg \& Sohn, Braunschweig, 1951.

23. Lalitha, G. \& Reddy, P.V. Elastic behavior of strontium-doped lanthanum calcium manganites in the vicinity of $T_{C} . J$. Phys. Chem. Solids 70, 960-966 (2009).

24. Abdulvagidov, Sh.B., Kamilov, I.K., Aliev, A.M. \& Batdalov, A.B. Heat capacity and electric resistance of $\mathrm{Sm}_{0.55} \mathrm{Sr}_{0.45} \mathrm{MnO}_{3}$ manganite near $T_{C}$ in a magnetic field of up to 26 kOe: fluctuation effects and colossal magnetoresistance development scenario. $Z h$. Éksp. Teor. Fiz. 123, 857 (2003) [JETP 96, 757 (2003)].

25. De Teresa, J.M. et al. Magnetic versus orbital polarons in colossal magnetoresistance manganites. Phys. Rev. B 65, 100403(R) (2002).

\section{Affiliations}

Institute of Physics of Dagestan Federal Research Centre of RAS, 94, Makhachkala

367015 Republic of Dagestan, Russian Federation

\section{Additional Information}

Reprints and permissions information is available online at www.nature.com/reprints. Correspondence and requests for materials should be addressed to the author.

\section{Competing financial interests}

The author declares no competing financial interests. 
Table I. | Initial data and fitted parameters in tracing the phase boundaries

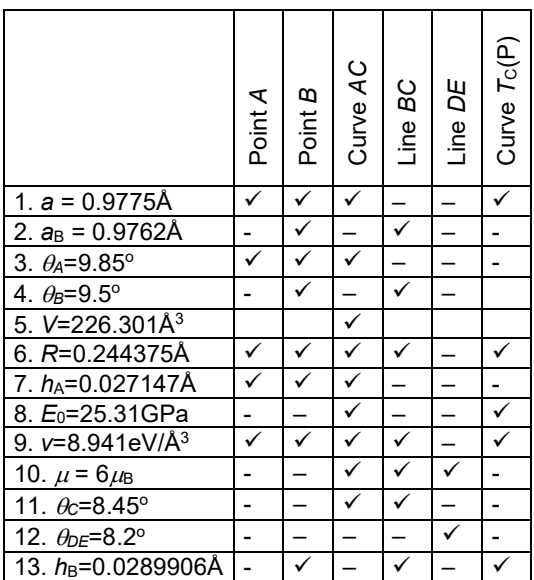

The data in rows $1-5$ are calculated from the neutron diffraction ${ }^{16,21}: a$ and $a_{\mathrm{B}}$ are half the $\mathrm{Mn}-\mathrm{O}$ distances before and after the transition; $\theta_{\mathrm{A}}$ and $\theta_{\mathrm{B}}$ are calculated as $\theta=\left(180^{\circ}-\alpha\right) / 2$ where the angle $\alpha=\mathrm{Mn}^{3+\mathrm{O}^{2}-} \mathrm{Mn}^{4+}$; and $V$ is a multiplication of the primitive-cell constants $5.424,7.678$ and

$5.434 \AA$. $R$ a fourth of the orbital length is standard for the $d$ and $p$

orbitals ${ }^{22} . h_{\mathrm{A}}, E_{0}$, and $v$ are fitted parameters of curve $A C$ (the normal-

condition Young's modulus $E_{0}$ is in good agreement with values for

manganites $\left.{ }^{23}\right) . \theta_{C}$ and $\theta_{D E}$ are fitted parameters of $A C$ and $D E$ lines.

\section{FIGURES LEGENDS}

